\title{
LONG TIME ASYMPTOTICS OF THE KORTEWEG-DE VRIES EQUATION
}

BY

STEPHANOS VENAKIDES

\begin{abstract}
We study the long time evolution of the solution to the Kortewegde Vries equation with initial data $v(x)$ which satisfy

$$
\lim _{x \rightarrow-\infty} v(x)=-1, \quad \lim _{x \rightarrow+\infty} v(x)=0 .
$$

We show that as $t \rightarrow \infty$ the step emits a wavetrain of solitons which asymptotically have twice the amplitude of the initial step. We derive a lower bound of the number of solitons separated at time $t$ for $t$ large.
\end{abstract}

1. Introduction. We study the long time behaviour of the solution $u(x, t)$ of the Korteweg-de Vries (KdV) equation

$$
u_{t}-6 u u_{x}+u_{x x x}=0
$$

with a step-like initial potential

$$
u(x, 0)=v(x), \quad \lim _{x \rightarrow+\infty} v(x)=0, \quad \lim _{x \rightarrow-\infty} v(x)=1 .
$$

Under general conditions, ensuring that the method of inverse scattering is applicable, Hruslov [1] proves that the step emits a wavetrain of solitons as $t \rightarrow \infty$. The solitons are asymptotically twice as high as the initial step and have a varying phase. We show here that if $N(t)$ denotes the number of solitons which have separated at time $t$, then

$$
N(t) \geqslant t^{1 / 4-\varepsilon} \quad \text { as } t \rightarrow+\infty .
$$

We use the method of inverse scattering to solve the $\mathrm{KdV}$ equation. Our derivation of the soliton wavetrain differs from Hruslov's in the fact that while he performs the asymptotic analysis starting at the level of the Marcenko kernel, we first solve the Marcenko equation and do the asymptotics on the explicit formula for $u(x, t)$.

We apply the inverse scattering theory for steplike potentials developed by Buslaev and Fomin [4], and studied rigorously by Cohen and Kappeller [2,3], who find necessary and sufficient conditions on a 2 by 2 matrix to be the scattering matrix of a steplike potential in a certain class. We assume $v(x)$ to satisfy

$$
\int_{0}^{\infty}|v(x)|(1+|x|) d x<\infty, \quad \int_{-\infty}^{0}|1+v(x)|(1+|x|) d x<\infty .
$$

Received by the editors April 10, 1985.

1980 Mathematics Subject Classification. Primary 35Q20, 35B40; Secondary 35P25, 35R30, 76B25.

${ }^{1}$ Research supported by the Office of Naval Research, the Air Force Office of Scientific Research, the Army Research Office, and the National Science Foundation. 
(ii) $v(x)$ has no bound states.

(iii) We neglect the reflection coefficient. Hruslov shows in [1] that this assumption, for the $(x, t)$ region we work in, is true asymptotically as $t \rightarrow \infty$.

Under these conditions the solution of the $\mathrm{KdV}$ equation is given by (see e.g. [1, equations (2.10-2.13)])

$$
u(x, t)=-2(\partial / \partial x) A(x, x),
$$

where $A(x, y)$ satisfies the Marcenko equation

$$
A(x, y)+G(x+y, t)+\int_{x}^{\infty} G(y+t, t) A(x, t) d t, \quad x<y,
$$

and the Marcenko kernel $G(x+y, t)$ is given by ( $\operatorname{set} c=1, \eta=\left(1-k^{2}\right)^{1 / 2}, S_{12}=0$ in [1, equation (2.10)])

$$
G(x+y, t)=\int_{0}^{1} P(\eta) e^{8 t \eta^{3}-\eta(x+y)} d \eta, \quad P(\eta) \geqslant 0, P(1)=0 .
$$

The function $P(\eta)$ constitutes the scattering data of the potential $v(x) . u(x, t)$ can be expressed explicitly in terms of the Marcenko kernel $G$ through Bargmann's Formula

$$
u(x, t)=2\left(\partial^{2} / \partial x^{2}\right) \log \Delta
$$

where

$$
\Delta(x, t)=\operatorname{det}(I+W(x, t))
$$

and

$$
W(x, t): L^{2}(0, \infty) \rightarrow L^{2}(0, \infty) \quad(x, t: \text { parameters }),
$$

Distr. Kernel of $W(x, t)=G(r+s+2 x, t)$.

The determinant is meant in the classical Fredholm sense.

Our normalizing the initial step to have height equal to 1 implies no loss of generality, since it is verified that if $u(x, t)$ satisfies the $\mathrm{KdV}$ equation so does $v(x, t)=c^{2} u\left(c x, c^{3} t\right)$.

Soliton wavetrains similar to the ones in question have been observed in experiments involving waves in a shallow canal generated by ships.

2. The explicit solution. We outline our method of computing

$$
\Delta(x, t)=\operatorname{det}(I+W)
$$

developed in [5]. The operator in (1.10),

$$
W: L^{2}(0, \infty) \rightarrow L^{2}(0, \infty),
$$

$$
\text { Distr. Ker. } W(x, t)=\int_{0}^{1} P(\eta) e^{8 \eta^{3} t-2 \eta x} e^{-\eta(r+s)} d \eta, \quad P(\eta) \geqslant 0,
$$

parametrized by $x$ and $t$, can be factored as

$$
W=\mathscr{L} \mathscr{M} \mathscr{L}=\left(\mathscr{L} \mathscr{M}^{1 / 2}\right)\left(\mathscr{M}^{1 / 2} \mathscr{L}\right),
$$

where $\mathscr{L}$ is the Laplace transform and $\mathscr{M}$ is multiplication by $P(\eta) e^{8 \eta^{3} t-2 \eta x}$.

$$
\left\|\mathscr{L} \mathscr{M}^{1 / 2}\right\|_{\text {Schimdt }}=\left\|\mathscr{M}^{1 / 2} \mathscr{L}\right\|_{\text {Schmidt }}=\int_{0}^{1} \int_{0}^{\infty} \frac{\mathscr{M}(\eta)}{(\eta+\mu)^{2}} d \mu d \eta .
$$


We assume

$$
\int_{0}^{1} \frac{\mathscr{M}(\eta)}{2 \eta}<\infty
$$

Hence by (2.2) $W$ belongs to the trace class and has the same eigenvalues as the operator

$$
K=\mathscr{M}^{1 / 2} \mathscr{L} \mathscr{L} \mathscr{M}^{1 / 2}=\mathscr{M}^{1 / 2} \mathscr{L}^{2} \mathscr{M}^{1 / 2}, \quad K: L^{2}(0, \infty) \rightarrow L^{2}(0, \infty),
$$

with the distribution kernel

$$
k(\eta, \mu)=\frac{\mathscr{M}^{1 / 2}(\eta, x, t) \mathscr{M}^{1 / 2}(\mu, x, t)}{(\eta+\mu)} .
$$

Clearly,

$$
\Delta(x, t)=\operatorname{det}(I+W)=\operatorname{det}(I+K) .
$$

We write the classical Fredholm series for $\operatorname{det}(I+K)$ and using the Cauchy determinant formula

$$
\operatorname{det}\left(\frac{1}{\eta_{i}+\eta_{j}}\right)_{i, j=1, \ldots, n}=\frac{\prod_{i \neq j}\left|\eta_{i}-\eta_{j}\right|}{\prod_{i, j}\left|\eta_{i}+\eta_{j}\right|}
$$

we obtain

$$
\Delta(x, t)=1+\sum_{m=1}^{\infty} d_{m}
$$

$$
d_{m}=\frac{1}{m !} \int_{0}^{1} \cdots \int_{0}^{1} \prod_{i=1}^{m} \frac{\mathscr{M}\left(\eta_{i}, x, t\right)}{2 \eta i} \prod_{1 \leqslant i<j \leqslant m}\left(\frac{\eta_{i}-\eta_{j}}{\eta_{i}+\eta_{j}}\right)^{2} d \eta_{1} \cdots d \eta_{m}
$$

This method is a natural generalization of the Kay-Moses determinant [6] and its expansion by Lax-Levermore [7]. We recall that

$$
\mathscr{M}(\eta, x, t)=P(\eta) e^{8 \eta^{3} t-2 \eta x}, \quad \int_{0}^{1}\left|\frac{P(\eta)}{\eta}\right| d \eta<\infty .
$$

Formula (2.7) can be generalized formally in three ways to give more solutions to the $\mathrm{KdV}$ equation.

(i) Allowing $x$ and $t$ to be complex.

(ii) Replacing the integration contour $[0,1]$ by some curve $C$ in the complex plane which does not contain both $\eta$ and $-\eta$ (otherwise $\left(\eta_{i}-\eta_{j}\right) /\left(\eta_{i}+\eta_{j}\right)$ will be singular). For example, if the curve $C$ is the line $z=\alpha+i y$ ( $\alpha=$ constant, $y$ real), we obtain as $\alpha \rightarrow 0$ a formula derived by McKean [8, p. 248] for initial data free of solitons. In McKean's formula $P(\eta)$ is the reflection coefficient at frequency $\eta$.

(iii) Regarding $P(\eta) d \eta$ as a complex measure on the curve $C$ satisfying

$$
d \mu=P(\eta) d \eta, \quad \int_{C} \frac{1}{|\eta|} d|\mu|<\infty .
$$

If we choose $\mu$ to be a positive atomic measure supported at $\eta=\eta_{0}>0$ we recover the simple soliton solution. 
The following estimate will show that after a certain index, the series for $\Delta(x, t)$ and its $x$ and $t$ derivatives converges rapidly.

THEOREM 2.1. Let

$$
\begin{gathered}
d_{m}^{(k, \lambda)}=\frac{\partial^{k+\lambda} d_{m}(x, t)}{\partial^{k} x \partial^{\lambda} t}, \\
\mathscr{M}(\eta, x, t)=P(\eta) e^{8 \eta^{3} t-2 \eta x}, \quad \int_{0}^{1} \frac{P(\eta)}{\eta} d \eta<\infty, \quad P(\eta) \geqslant 0 .
\end{gathered}
$$

Then

$$
\left|\frac{d_{m+1}^{(k, \lambda)}}{d_{m}^{(k, \lambda)}}\right| \leqslant 2^{k+\lambda} \int_{0}^{1}\left|\frac{\mathscr{M}(\eta)}{2 \eta}\right| e^{-2 m \eta} d \eta
$$

Proof. By symmetry,

$$
d_{m}^{(k, \lambda)}=\frac{1}{m !} \int_{0}^{1} \cdots \int_{0}^{1} \cdot d^{m} \eta=\int_{0}^{1} \cdots \int_{0}^{\eta_{3}} \int_{0}^{\eta_{2}} \cdot d \eta_{1} d \eta_{2} \cdots d \eta_{m}
$$

Differentiation by $x$ or $t$ lowers the factor $-2 \sum_{j=1}^{m} \eta_{j}$ or $8 \sum_{j=1}^{m} \eta_{j}^{3}$, respectively.

$$
\begin{gathered}
0 \leqslant \eta_{1} \leqslant \eta_{j} \Rightarrow \sum_{j=1}^{m} \eta_{j} \leqslant 2 \sum_{j=2}^{m} \eta_{j}, \quad \sum_{j=1}^{m} \eta_{j}^{3} \leqslant 2 \sum_{j=2}^{m} \eta_{j}^{3}, \\
0 \leqslant \eta_{1} \leqslant \eta_{j} \leqslant 1 \Rightarrow\left|\frac{\eta_{1}-\eta_{j}}{\eta_{1}+\eta_{j}}\right|=\left|\frac{1-\eta_{j} / \eta_{1}}{1+\eta_{j} / \eta_{1}}\right| \leqslant e^{-\eta_{1} / \eta_{j}} \leqslant e^{-\eta_{1}}, \\
d_{m+1}^{(k, \lambda)}=\int_{0}^{1} \cdots \int_{0}^{\eta_{3}}\left[\int_{0}^{\eta_{2}} \frac{\mathscr{M}\left(\eta_{1}\right)}{2 \eta_{1}} \prod_{j=2}^{m+1}\left(\frac{\eta_{1}-\eta_{j}}{\eta_{1}+\eta_{j}}\right)^{2} d \eta_{1}\right]\left(\sum_{j=1}^{m+1}\left(-2 \eta_{j}\right)\right)^{k} \\
\cdot\left(\sum_{j=1}^{m+1} 8 \eta_{j}^{3}\right)^{\lambda} \prod_{j=2}^{m+1} \frac{\mathscr{M}\left(\eta_{j}\right)}{2 \eta_{j}} \prod_{2 \leqslant i<j \leqslant m+1}\left(\frac{\eta_{i}-\eta_{j}}{\eta_{i}+\eta_{j}}\right)^{2} d \eta_{2} \cdots d \eta_{m+1} .
\end{gathered}
$$

By (2.7b) and the above estimates

$$
\left|d_{m+1}^{(k, \lambda)}\right| \leqslant\left|d_{m}^{(k, \lambda)}\right| 2^{k+\lambda} \int_{0}^{1} \frac{\mathscr{M}(\eta)}{2 \eta} e^{-2 m \eta} d \eta \text {. Q.E.D. }
$$

3. Long time asymptotics. We have

$$
u=-2 \frac{\partial^{2}}{\partial x^{2}} \log \Delta(x, t), \quad \Delta(x, t)=1+\sum_{m=1}^{\infty} d_{m}(x, t),
$$

with $d_{m}$ as in (2.7).

Lemma 3.1. Let $x \geqslant 4 t$. Then $\lim _{t \rightarrow \infty} u(x, t)=0$ uniformly in $x$. 
Proof. $x \geqslant 4 t \Rightarrow 8 \eta^{3} t-2 \eta x \leqslant-8 t \eta\left(1-\eta^{2}\right)$. Let

$$
d_{m}^{(k)}(x, t)=d_{m}^{(k, 0)}=\frac{\partial^{k} d_{m}}{\partial x^{k}}
$$

Eq. $(2.76) \Rightarrow d_{1}^{(k)}(x, t) \leqslant \int_{0}^{1} \frac{\mathscr{M}(\eta)}{2 \eta} d \eta=\int_{0}^{1} \frac{P(\eta)}{2 \eta} e^{-8 t \eta\left(1-\eta^{2}\right)} d \eta \rightarrow 0 \quad(t \rightarrow \infty)$.

Applying Theorem 2.1,

$$
\begin{aligned}
& \lim _{t \rightarrow \infty} \Delta(x, t)=1, \quad \lim _{t \rightarrow \infty} \frac{\partial^{k} \Delta(x, t)}{\partial x^{k}}=0 \quad \text { uniformly in } x \geqslant 4 t \\
& \lim _{t \rightarrow \infty} u(x, t)=\lim _{t \rightarrow \infty} \frac{-2\left(\Delta \Delta_{x}-\Delta_{x}^{2}\right)}{\Delta^{2}}=0 \quad \text { uniformly in } x \geqslant 4 t . \quad \text { Q.E.D. }
\end{aligned}
$$

DeFinition 3.2. Let $\Gamma(\lambda)$ be the rectangle of the $x-t$ plane defined by (3.2a) $\Gamma(\lambda)=\{(x, t): x=4 \lambda-\xi, t=\lambda+\tau, 0 \leqslant \xi \leqslant \gamma(\lambda),|\tau| \ll \log \lambda\}$ where $\gamma(\lambda)$ satisfies

$$
\gamma(\lambda) \ll \lambda^{1 / 4}, \quad \gamma(\lambda) \gg \log \lambda, \quad \text { as } \lambda \rightarrow \infty .
$$

We write the solution $u(x, t)$ in compact form:

$$
\begin{array}{cc}
\text { (3.3a-b) } u(x, t)=-2 \frac{\partial^{2}}{\partial x^{2}} \log \Delta(x, t), \quad \Delta(x, t)=1+\sum_{m=1}^{\infty} d_{m}(x, t), \\
\text { (3.3c) } & d_{m}(x, t)=\frac{1}{m !} \int_{0}^{1} \cdots \int_{0}^{1} e^{\Phi_{m}(\eta, \xi, \tau, \lambda)} G_{m}(\eta) d^{m} \eta
\end{array}
$$

where we have set

$$
\begin{gathered}
\Phi_{m}(\eta, \xi, \tau, \lambda)=\sum_{j=1}^{m}\left(-8 \lambda \eta_{j}\left(1-\eta_{j}^{2}\right)+2 \eta_{j} \xi+8 \eta_{j}^{3} \tau+\rho\left(\eta_{j}\right)\right), \\
G_{m}(\eta)=\prod_{\substack{i \neq j \\
1 \leqslant i, j \leqslant m}}\left|\frac{\eta_{i}-\eta_{j}}{\eta_{i}+\eta_{j}}\right|, \quad \rho\left(\eta_{j}\right)=\log \frac{P\left(\eta_{j}\right)}{\eta_{j}} .
\end{gathered}
$$

Obviously $0 \leqslant G_{m}(\eta) \leqslant 1$.

\section{LEMMA 3.3.}

$$
(x, t) \in \Gamma(\lambda) \Rightarrow \sum_{m=1}^{\infty} d_{m}^{(k)}(x, t) \sim \sum_{m \leqslant[\gamma]} d_{m}^{(k)}(x, t) \quad \text { as } \lambda \rightarrow \infty
$$

where $[\gamma]$ is the integer part of $\gamma$.

Proof. By Theorem 2.1 when $m \geqslant \gamma \geqslant \xi$,

$$
\begin{aligned}
\left|\frac{d_{m+1}^{(k)}}{d_{m}^{(k)}}\right| & \leqslant 2^{k} \int_{0}^{1} \exp \left[-8 \lambda \eta\left(1-\eta^{2}\right)+2 \eta \xi+8 \eta^{3} \tau-2 \eta m+\rho(\eta)\right] d \eta \\
& \leqslant 2^{k} \int_{0}^{1} \exp \left[-8 \lambda \eta\left(1-\eta^{2}\right)+8 \eta^{3} \log \lambda+\rho(\eta)\right] d \eta \rightarrow 0 \quad \text { as } \lambda \rightarrow \infty, \\
\sum_{m>[\gamma]} d_{m}^{(k)} \ll d_{[\gamma]}^{(k)} & (\lambda \rightarrow \infty) . \text { Q.E.D. }
\end{aligned}
$$


Now that we have truncated series (3.3b), we compute the integral (3.3c) for $d_{m}$ by showing that the main contribution comes from the vertices of the $m$-cube.

Definition 3.4. Let $d_{m}(x, t)=v_{m}(x, t)+r_{m}(x, t)$ where $v_{m}(x, t)$ is the contribution to integral (2.7b) from points $\left(\eta_{1}, \ldots, \eta_{m}\right)$ satisfying

$$
\text { either } 0 \leqslant \eta_{j} \leqslant \gamma^{2} / \lambda \text { or } 0 \leqslant 1-\eta_{j} \leqslant \gamma^{2} / \lambda \text {. }
$$

$r_{m}(x, t)$ is the contribution from the complement of the $m$-dimensional unit cube. We remind the reader that superscript ${ }^{(k)}$ denotes differentiation $k$ times by $x$, and that $\gamma^{2} / \lambda \rightarrow 0$ as $\lambda \rightarrow \infty$.

\section{LEMMA 3.5 .}

$$
(x, t) \in \Gamma(\lambda) \Rightarrow \sum_{m=1}^{[\gamma]} r_{m}^{(k)}(x, t) \rightarrow 0 \quad \text { as } \lambda \rightarrow \infty .
$$

Proof. One of the coordinates of point $\left(\eta_{1}, \ldots, \eta_{m}\right)$ contributing to $r_{m}(x, t)$, say $\eta_{j_{0}}$, satisfies $\gamma^{2} / \lambda \leqslant \eta_{j_{0}} \leqslant 1-\gamma^{2} / \lambda$. A fortiori,

$$
\sum_{j=1}^{m} \eta_{j}\left(1-\eta_{j}^{2}\right) \geqslant \eta_{j_{0}}\left(1-\eta_{j_{0}}^{2}\right) \geqslant \frac{\gamma^{2}}{2 \lambda} \text {. }
$$

Furthermore,

$$
\begin{gathered}
\sum_{j=1}^{m} \eta_{j} \leqslant m, \quad \sum_{j=1}^{m} \eta_{j}^{3} \leqslant m, \\
r_{m}^{(k)}(x, t) \leqslant \frac{2^{k} m^{k}}{m !} \int_{0}^{1} \cdots \int_{0}^{1} \exp \left[-8 \lambda \frac{\gamma^{2}}{2 \lambda}+2 \xi m+8 \tau m+\sum_{j=1}^{m} \rho\left(\eta_{j}\right)\right] d^{m} \eta .
\end{gathered}
$$

However,

$$
\begin{gathered}
\xi, m \leqslant \gamma(\lambda) \rightarrow \infty \text { as } \lambda \rightarrow \infty,|\tau| \leqslant \log \lambda, \\
r_{m}^{(k)}(x, t) \leqslant \frac{2^{k} m^{k}}{m !} e^{-2 \gamma^{2}+8 \gamma \log \lambda}\left\{\int_{0}^{1} e^{\rho(\eta)} d \eta\right\}^{m}, \\
\sum_{m=1}^{[\gamma]} r_{m}^{(k)}(x, t) \leqslant 2^{k} e^{-2 \gamma^{2}+8 \gamma \log \lambda} \sum_{m=1}^{\infty} \frac{m^{k}}{m !}\left(\int_{0}^{1} e^{\rho(\eta)} d \eta\right)^{m} \rightarrow 0 \text { as } \lambda \rightarrow \infty \text { Q.E.D. }
\end{gathered}
$$

We examine the contribution to $d_{m}(x, t)$ from the vertex having $\sigma$ ones and $\tau$ zeroes. By the symmetry of the integrand the order of 1's and 0 's does not matter. We decouple the integral (3.3c) to a product of a $\sigma$-tuple integral for the variables near 1 and a $\tau$-tuple integral for the variables near 0 by the following calculation:

Lemma 3.6. Let $0 \leqslant 1-\eta_{i} \leqslant \gamma^{2} / \lambda, i=1, \ldots, \sigma \leqslant m$, and $0 \leqslant \mu_{j} \leqslant \gamma^{2} / \lambda, j=$ $1, \ldots, \tau=m-\sigma$. If $m \leqslant \gamma(\lambda)<<\lambda^{1 / 4}$ as $\lambda \rightarrow \infty$, then

$$
\prod_{\substack{1 \leq i \leq \sigma \\ 1 \leqslant j \leqslant \tau}}\left|\frac{\eta_{i}-\mu_{j}}{\eta_{i}+\mu_{j}}\right| \sim 1 \quad \text { as } \lambda \rightarrow \infty \text {. }
$$

ProOF.

$$
1 \geqslant \prod\left|\eta_{i}-\mu_{j}\right| \geqslant\left(1-\frac{2 \gamma^{2}}{\lambda}\right)^{\sigma \tau} \geqslant e^{-\gamma^{2} \sigma \tau / \lambda} \geqslant e^{-\gamma^{4} / \lambda} \sim 1 \quad \text { as } \lambda \rightarrow \infty .
$$


The last relation is true since $\gamma^{4}<<\lambda$ by hypothesis. Similarly, $\Pi\left|\eta_{i}+\mu_{j}\right| \sim 1$ as $\lambda \rightarrow \infty$. Q.E.D.

We write $\Delta^{(k)}(x, t)$ asymptotically as the sum of the contributions from each vertex of each $m$-cube.

Definition 3.7. Let $\Phi_{\sigma}, G_{\sigma}$ be as in (3.3).

$$
\begin{gathered}
I_{\sigma}=\int_{1-\gamma^{2} / \lambda}^{1} \cdots \int_{1-\gamma^{2} / \lambda}^{1} e^{\Phi_{\sigma}(\eta, \xi, \tau, \lambda)} G_{\sigma}(\eta) d^{\sigma} \eta, \\
J_{\nu}=\int_{0}^{\gamma^{2} / \lambda} \cdots \int_{0}^{\gamma^{2} / \lambda} e^{\Phi_{\nu}(\eta, \xi, \tau, \lambda)} G_{\nu}(\eta) d^{\nu} \eta .
\end{gathered}
$$

By Lemmas 3.3, 3.5, and 3.6,

$$
\Delta(x, t) \sim 1+\sum_{\sigma=1}^{[\gamma]} \frac{I_{\sigma}}{(\sigma+\tau) !} \sum_{r=0}^{[\gamma]-\sigma}\left(\begin{array}{c}
\sigma+\tau \\
\tau
\end{array}\right) J_{\tau}=1+\sum_{\sigma=1}^{[\gamma]} \frac{I_{\sigma}}{\sigma !} \sum_{\tau=0}^{[\gamma]-\sigma} \frac{J_{\tau}}{\tau !} .
$$

LEMMA 3.8.

$$
\sum_{\nu=0}^{\infty} \frac{J_{\nu}}{\nu !} \sim 1, \quad \sum_{\nu=0}^{\infty} \frac{J_{\nu}^{(k)}}{\nu !} \rightarrow 0 \quad \text { as } \lambda \rightarrow \infty .
$$

Proof. $J_{0}=1$. When $\nu \geqslant 1,0 \leqslant \eta_{j} \leqslant \gamma^{2} / \lambda$. We have $0 \leqslant G_{\nu}(\eta) \leqslant 1$,

$$
\begin{aligned}
\Phi_{\nu}(\eta, \xi, \tau, \lambda) & =\sum_{j=1}^{\nu}\left(-8 \lambda \eta_{j}\left(1-\eta_{j}^{2}\right)+2 \xi \eta_{j}+8 \eta_{j}^{3} \tau+\rho\left(\eta_{j}\right)\right) \\
& \leqslant \sum_{j=1}^{\nu}\left(-4 \lambda \eta_{j}+2 \xi \eta_{j}+8 \eta_{j} \tau+\rho\left(\eta_{j}\right)\right) .
\end{aligned}
$$

Recalling $\xi \leqslant \gamma \leqslant \lambda^{1 / 4},|\tau| \leqslant \log \lambda$,

$$
\Phi_{\nu}(\eta, \xi, \tau, \lambda) \leqslant-\frac{\lambda}{2} \sum_{j=1}^{\nu} \eta_{j}+\sum_{j=1}^{\nu} \rho\left(\eta_{j}\right) .
$$

From (3.6),

$$
J_{\nu} \leqslant\left[\int_{0}^{1} e^{-(\lambda / 2) \eta_{j}+\rho\left(\eta_{j}\right)} d \eta_{j}\right]^{\nu}
$$

The result follows immediately (estimates for $J_{\nu}^{(k)}$ are identical). Q.E.D.

Substituting in (3.7) we obtain

$$
\Delta(x, t) \sim 1+\sum_{\sigma=1}^{[\gamma]} \frac{I_{\sigma}}{\sigma !} \quad \text { as } \lambda \rightarrow \infty .
$$

By our previous estimates it is clear that this relation can be differentiated term by term.

THEOREM 3.9. Let the scattering data of the initial potential (see (1.6), (1.7)) satisfy

$$
e^{\rho(\eta)}=\frac{P(\eta)}{\eta} \sim K(1-\eta)^{\alpha}, \quad \alpha>-1 \quad \text { as } \eta \rightarrow 1
$$

Then

$$
\Delta(x, t) \sim 1+\sum_{m=1}^{[\gamma]} A_{m} \lambda^{-m^{2}-\alpha m} e^{2(\xi+4 \tau) m},
$$


where

$$
A_{m}=\frac{1}{m ! 2^{m^{2}-m}} \int_{0}^{\infty} \cdots \int_{0}^{\infty} \prod_{j=1}^{m} p_{j}^{\alpha} e^{-16 p_{j}} \prod_{\substack{i, j=1 \\ i \neq j}}^{m}\left|p_{i}-p_{j}\right| d^{m} p .
$$

Proof. In (3.8) we calculate $I_{\sigma}$ defined by (3.5). We make the change of variable

$$
1-\eta_{j}=p_{j} / \lambda \text {. }
$$

$1-\gamma^{2} / \lambda \leqslant \eta_{j} \leqslant 1$ is transformed to $0 \leqslant p_{j} \leqslant \gamma^{2}$;

$$
\prod_{\substack{i, j=1 \\ i \neq j}}^{m}\left|\eta_{i}+\eta_{j}\right|=\prod_{\substack{i, j=1 \\ i \neq j}}^{m}\left|2-\frac{\left(p_{i}+p_{j}\right)}{\lambda}\right| \sim 2^{m(m-1)} \text { as } \lambda \rightarrow \infty .
$$

An elementary calculation using (3.9) gives that as $\lambda \rightarrow \infty$ :

$$
I_{m}(x, t) \sim\left(\frac{1}{\lambda}\right)^{m^{2}+\alpha m} \frac{e^{2 \xi m+8 \tau m}}{2^{m(m-1)}} \int_{0}^{\gamma^{2}} \cdots \int_{0}^{\gamma^{2}} \prod_{j=1}^{m}\left(p_{j}^{\alpha} e^{-16 p_{j}}\right) \prod_{\substack{i, j=1 \\ i \neq j}}^{m}\left|p_{i}-p_{j}\right| d^{m} p .
$$

We substitute in (3.8) and can replace the upper limits of integration by $\infty$ to obtain the result. Relation (3.10a) can clearly be differentiated term by term in $\xi$ and $\tau$.

4. The soliton wavetrain. We obtained in the previous section

(3.10) $\Delta(x, t) \sim 1+\sum_{m=1}^{[\gamma]}\left[\exp \left(-m^{2}-\alpha m\right) \log \lambda+2(\xi+4 \tau) m+\log A_{m}\right]$.

To focus onto the solitons we let

$$
\xi=\left(N+\frac{\alpha}{2}+\frac{1}{2}\right) \log \lambda-\zeta, \quad N=0,1,2, \ldots,[\gamma(\lambda) / \log \lambda],
$$

We are looking into the regions (see (3.2a))

$$
x_{N}=4 \lambda-\left(N+\frac{\alpha}{2}+\frac{1}{2}\right) \log \lambda+\zeta, \quad \zeta<\log \lambda .
$$

Substituting (3.11) into (3.10) we obtain

$$
\begin{aligned}
\Delta\left(x_{N}, t\right) \sim 1+\sum_{m=1}^{[\gamma]} \exp \left\{\operatorname { l o g } \lambda \left[-m^{2}\right.\right. & +(2 N+1) m] \\
& \left.+\log A_{m}-2(\zeta-4 \tau) m\right\},
\end{aligned}
$$

$$
\begin{aligned}
\Delta^{(k)}\left(x_{N}, t\right) \sim \sum_{m=1}^{[\gamma]}(-2)^{k} m^{k} \exp \{\log \lambda & {\left[-m^{2}+(2 N+1) m\right] } \\
& \left.+\log A_{m}-2(\zeta-4 \tau) m\right\} .
\end{aligned}
$$

Differentiation of the asymptotic relation is possible because all our calculations hold for derivatives of $d_{m}(x, t)$ as well. Series (3.13) is dominated by the terms that maximize $\left\{-m^{2}+(2 N+1) m\right\}$. This happens when $m=N$ and when $m=N+1$.

$$
\max _{m \in Z}\left\{-m^{2}+(2 N+1) m\right\}=N^{2}+N \text {. }
$$


Hence,

$$
\begin{aligned}
\Delta\left(x_{N}, t\right) \sim & \exp \left[\left(N^{2}+N\right) \log \lambda-2(\zeta-4 \tau) N+\log A_{N}\right] \\
& \cdot\left[1+\exp [-2(\zeta-4 \tau)+\underbrace{\log \frac{A_{N+1}}{A_{N}}}_{2 \phi_{N}}]\right] .
\end{aligned}
$$

(3.14a) can be written in the form

$$
\Delta\left(x_{N}, t\right) \sim e^{f(\lambda, \tau) \zeta+g(\lambda, \tau)} \cosh \left[\zeta-4 \tau-\phi_{N}\right] .
$$

By (1.8),

$$
\begin{aligned}
& u\left(x_{N}, t\right)=-2 \frac{\partial^{2}}{\partial x_{N}^{2}} \Delta\left(x_{N}, t\right)=-2 \frac{\partial^{2}}{\partial \zeta^{2}} \Delta\left(x_{N}, t\right), \\
& u\left(x_{N}, t\right) \sim-2 \operatorname{sech}^{2}\left[\zeta-4 \tau-\phi_{N}\right]=\text { soliton. }
\end{aligned}
$$

In the region between two solitons it is easy to show that $u(x, t) \rightarrow 0$. In particular, $\Delta(x, t)$ is dominated by just one term of the form $e^{f(\lambda, \tau) \zeta+g(\lambda, \tau)}$, hence

$$
\partial^{2} / \partial \zeta^{2} \log \Delta \rightarrow 0
$$

We can now return to $x, t$ variables. Setting $\tau=0$ gives $t=\lambda, x_{N}=4 t-$ $\left(N+\frac{1}{2}\right) \log t+\zeta$.

We have shown

THEOREM 4.1. Let the step-like function $v(x)$ satisfy conditions (1.4) and (3.9). Let $u(x, t)$ solve the I.V.P.

$$
u_{t}-6 u u_{x}+u_{x x x}=0, \quad u(x, 0)=v(x) .
$$

As $t \rightarrow \infty$ and in a region $x \geqslant 4 t-\gamma(t)$ where $\gamma(t)<<t^{1 / 4}$ we have

$$
u(x, t) \sim \sum_{N=1}^{[\gamma] / \log t}-2 \operatorname{sech}^{2}\left[x-4 t+\left(N+\frac{\alpha}{2}+\frac{1}{2}\right) \log t-\frac{1}{2} \log \frac{A_{N+1}}{A_{N}}\right]
$$

where $A_{N}$ is given by (3.10b).

\section{REFERENCES}

1. E. Ja. Hruslov, Asymptotics of the solution of the Cauchy problem for the Korteweg-de Vries equation with initial data of step type, Math USSR-Sb. 28 (1976).

2. A. Cohen and T. Kappeler, Scattering and inverse scattering for step-like potentials, The Schrödinger Equation, 1983, Preprint.

3. A. Cohen, Solutions of the Korteweg-de Vries equation with steplike initial profile, 1983, Preprint.

4. V. S. Buslaev and V. N. Fomin, An inverse scattering problem for the one dimensional Schrödinger equation on the entire axis, Vestnik Leningrad Univ. 17 (1962), 56-64.

5. S. Venakides, The zero dispersion limit of the Korteweg-de Vries equation with non-trivial reflection coefficient, Comm. Pure Appl. Math. 38 (1985), 125-155.

6. I, Kay and H. E. Moses, J. Appl. Phys. 27 (1956), 1503-1508.

7. P. D. Lax and C. D. Levermore, The small dispersion limit of the Korteweg-de Vries equation. I, II, Comm. Pure Appl. Math. 36 (1983), 253-290; Comm. Pure Appl. Math. 36 (1983), $571-593$.

8. H. P. McKean, Theta functions, solitons and singular curves, Partial Differential Equations and Geometry, Proceedings of the Park City Conference (Christopher Byrnes, ed.), Marcel Dekker, New York, 1979. 